\title{
IMPROVING GENE EXPRESSION CANCER MOLECULAR PATTERN DISCOVERY USING NONNEGATIVE PRINCIPAL COMPONENT ANALYSIS
}

\author{
XIAOXU HAN \\ xiaoxu.han@emich.edu
}

\begin{abstract}
Department of Mathematics and Bioinformatics Program, Eastern Michigan University, Ypsilanti MI, 48197 USA
\end{abstract}

\begin{abstract}
Robust cancer molecular pattern identification from microarray data not only plays an essential role in modern clinic oncology, but also presents a challenge for statistical learning. Although principal component analysis (PCA) is a widely used feature selection algorithm in microarray analysis, its holistic mechanism prevents it from capturing the latent local data structure in the following cancer molecular pattern identification. In this study, we investigate the benefit of enforcing non-negativity constraints on principal component analysis ( $\mathrm{PCA}$ ) and propose a nonnegative principal component (NPCA) based classification algorithm in cancer molecular pattern analysis for gene expression data. This novel algorithm conducts classification by classifying meta-samples of input cancer data by support vector machines (SVM) or other classic supervised learning algorithms. The meta-samples are low-dimensional projections of original cancer samples in a purely additive meta-gene subspace generated from the NPCA-induced nonnegative matrix factorization (NMF). We report strongly leading classification results from NPCA-SVM algorithm in the cancer molecular pattern identification for five benchmark gene expression datasets under 100 trials of $50 \%$ hold-out cross validations and leave one out cross validations. We demonstrate superiority of NPCA-SVM algorithm by direct comparison with seven classification algorithms: SVM, PCA-SVM, KPCASVM, NMF-SVM, LLE-SVM, PCA-LDA and $k-N N$, for the five cancer datasets in classification rates, sensitivities and specificities. Our NPCA-SVM algorithm overcomes the over-fitting problem associative with SVM-based classifications for gene expression data under a Gaussian kemel. As a more robust high-performance classifier, NPCA-SVM can be used to replace the general SVM and k-NN classifiers in cancer biomarker discovery to capture more meaningful oncogenes.
\end{abstract}

Keywords: Nonnegative principal component analysis (NPCA)

\section{Introduction}

With the recent development of genomics and proteomics, molecular diagnostics has appeared as a novel tool to diagnose cancers. It picks a patient's tissue, serum or plasma samples and uses DNA chips or mass spectrometry (MS) based proteomics techniques to generate gene/protein expressions of these samples. The gene/protein expressions reflect gene/protein activity patterns in different types of cancerous or precancerous cells: i.e., they are molecular patterns or molecular signatures of cancers. Different cancers have different molecular patterns and the molecular patterns of a normal cell will be different from those of a cancer cell. In modern oncology, clinicians more and more rely on the robust classifications of gene/protein expression patterns to identify cancerous tissues and find their corresponding biomarkers. However, it is still a challenge for oncologists and computational biologists to robustly classify cancer molecular patterns because of the special characteristics of gene/protein expression data. In this study, we mainly focus on the cancer molecular pattern identification for gene expression data. 
Gene expression data are characterized by high dimensionalities. It can be represented by a $n \times m$ matrix after preprocessing. Each row represents the expression levels of a gene across different biological samples; each column represents the gene expression levels of a genome under a sample. Usually $n \gg m$, i.e., the number of variables (genes) is much greater than the number of biological samples. The number of samples is $<200$ and the number of genes $>5000$ generally. These data are not noise-free because their raw data contain a large amount of systematic noise and preprocessing algorithms can not remove them completely. Although there are a large amount of variables in these data, only a small set of variables have meaningful contributions to data variations.

Many feature selection algorithms are employed to reduce gene expression data dimensions before further classification/clustering analysis [1,2,3,4]. Principal component analysis (PCA) may be the mostly used approach among them. It projects data in an orthogonal subspace generated by the eigenvectors of a data covariance or correlation matrix. The data representation in the subspace is uncorrelated and the maximum variance directions based subspace spanning guarantees the least information loss in feature selection. However, as a holistic feature selection algorithm, PCA can only capture the features contributing to the global characteristics of data and miss the features contributing to the local characteristics of data. This holistic feature selection mechanism not only leads to the hard time to interpret each principal component (PC) intuitively, but also hurdles the subtle data local latent structure discovery in the following clustering/ classification, because each PC only contains some levels of global characteristics of data.

One important reason for the holistic mechanism in PCA is that data representation in classic PCA is not 'purely additive', i.e., linear combinations in the PCA mix with both positive and negative weights and each $\mathrm{PC}$ consists of both negative and positive entries. The positive and negative weights are likely to cancel each other partially in data representation. Actually, it is more likely that weights contributing to local features are cancelled out than weights contributing to global features in linear combinations. This mainly leads to the data locality loss and holistic feature selection characteristics in PCA.

Imposing nonnegative constraints on PCA, i.e., restricting the signs of all entries of the PC matrix $U$ to be nonnegative, can remove the likelihood of partial-cancellations and make data representation consisting of only additive components. It also contributes to the intuitive interpretation and sparse representation of each PC. In the context of feature selection, adding nonnegative constraints on PCA can improve data locality in feature selection and make data latent structure explicitly. Actually, adding non-negativity on PCA is actually also motivated by cancer molecular pattern discovery itself. Gene expression data generally are represented as positive or nonnegative matrices naturally or after simple processing. It is reasonable to require their corresponding dimension reduction data to be positive or at least nonnegative to maintain data locality in feature selection and for the sake of following clustering /classification.

\section{Nonnegative Principal Component Analysis (NPCA)}

Nonnegative PCA can be viewed as an extension of classic PCA by imposing PCA with non-negativity constraints in order to capture data locality in feature selection. 
Let $X=\left(x_{1}, x_{2}, \cdots x_{n}\right), x_{i} \in \Re^{d}$, be a zero mean dataset, i.e., $\sum_{i=1}^{n} x_{i}=0$, a nonnegative PCA can be formulated as a constrained optimization problem to find maximum variance directions under nonnegative constraints. For instance, the first nonnegative maximum variance direction $u$ i.e., $1^{\text {st }}$ nonnegative $\mathrm{PC}$ can be found by solving the following optimization problem:

$$
\begin{aligned}
& \max u^{T} C u, \quad \text { s.t. } \\
& u^{T} u=1, u \geq 0
\end{aligned}
$$

Where $C=\frac{1}{n} X X^{T}$ is the covariance matrix of the input dataset $X$. If $X$ is not a zero mean data, the data covariance matrix can be estimated by the equation: $C=\frac{1}{n}\left(X X^{T}-\frac{1}{n} X \overrightarrow{11}^{T} X^{T}\right)$, where $\overrightarrow{1} \in \Re^{d}$ with all entries are '1's. Similarly, all nonnegative maximum variance directions can be found by solving the optimization problem,

$$
\begin{aligned}
& \max J(U)=\frac{1}{2}\left\|U^{T} X\right\|_{F}^{2}, \quad \text { s.t. } \\
& U^{T} U=I, U \geq 0
\end{aligned}
$$

Where $U=\left[u_{1}, u_{2}, \cdots u_{k}\right], k \leq d$, is a set of nonnegative PCs. In fact, the rigorous orthonormal constraint under non-negativity is too strict for practical cancer molecular pattern analysis. Because it requires only one nonnegative entry in each column of $U$, i.e., each PC only contains one nonnegative entry. However, such a constraint is not congruent to the fact that the expressions of many key genes can be involved in developing the cancerous cells. This fact requires that each corresponding PC has more than one nonnegative entry. That is, the quadratic programming problem with the orthonormalnonnegativity condition can be further relaxed as,

$$
\max _{U \geq 0} J(U, \alpha)=\frac{1}{2}\left\|U^{T} X\right\|_{F}^{2}-\alpha\left\|I-U^{T} U\right\|_{F}^{2}
$$

Where $\alpha \geq 0$ is a parameter to control the orthonormal degree of each column of $U$. After relaxation, matrix $U$ is a near-orthonormal nonnegative matrix, i.e., $U^{T} U \sim I$. We give a gradient learning method for this nonlinear optimization problem as follows. Computing the gradient of the objective function with respective to $U$, we have

$$
U(t+1)=U(t)-\eta(t) \nabla_{U} J(t), U \geq 0
$$

Where $\nabla_{U} J(U, \alpha)=\left(U^{T} X\right) X^{T}+2 \alpha\left(I-U^{T} U\right) U$ and $\eta(t)$ is the iteration step size in the $\mathrm{t}$ time level iteration. For convenience, we select the step size in the iteration as 1 . This is equivalent to finding the local maximum of the following function $f\left(u_{s l}\right)$ in the equation (5) under the conditions: $u_{s l} \geq 0$, where $s=1,2 \cdots d ; l=1,2 \cdots n$ in the scalar level. 


$$
\max _{u_{s l} \geq 0} f\left(u_{s l}\right)=-\alpha u_{s l}^{4}+c_{2} u_{s l}^{2}+c_{1} u_{s l}+c_{0}
$$

Where $c_{2}$ and $c_{1}$ are the coefficients of $u_{s l}^{2}$ and $u_{s l} ; c_{0}$ is the sum of the constant items independent of $u_{s l}$. Computing stationary points for $f\left(u_{s l}\right)$, we have a cubic function root finding problem. The final $U$ matrix is a set of nonnegative roots of equation (6).

$$
p\left(u_{s l}\right)=d f\left(u_{s l}\right) / d u_{s l}=-4 \alpha u_{s l}^{3}+2 c_{2} u_{s l}+c_{1}=0
$$

By collecting the coefficients of $u_{s l}$ and $u_{s l}^{2}$, we have

$$
\begin{aligned}
& c_{2}=\frac{1}{2} \sum_{i=1}^{n} x_{s i}^{2}-\alpha \sum_{j=1, j \neq l}^{k} u_{s j}^{2}-2 \alpha \sum_{t=1, t \neq s}^{d} u_{t l}^{2}+2 \alpha \\
& c_{1}=\sum_{i=1}^{n} \sum_{t=1, t \neq s}^{d} x_{s i} u_{t l} x_{t i}-2 \alpha \sum_{j=1, j \neq i}^{k} \sum_{t=1, t \neq s}^{d} u_{s j} u_{t l} u_{t j}
\end{aligned}
$$

Actually, the constant term $c_{0}=-k \alpha$ does not have affects on the entries of $U$ matrix. Only $c_{1}$ and $c_{2}$ are involved in the nonnegative root finding of equation (6). The complexity for the nonnegative principal component analysis algorithm (NPCA) is $O(d k n \times N)$, where $N$ is the total iteration number in the algorithm to achieve the final termination threshold.

\section{NPCA-based Cancer Molecular Pattern Classification}

The nonnegative principal component analysis (NPCA) based cancer molecular pattern classification first employs NPCA to obtain the nonnegative representation of each biological sample in a purely additive low dimensional subspace spanned by meta-genes. A meta-gene is a linear combination of the expression levels of all genes in a cancer dataset. The nonnegative representation of a biological sample in NPCA is a metasample, which is the prototype of the sample with small dimensionalities. Then, a classification algorithm $\pi_{A}$, which can be any classification algorithm, is applied to the meta-samples to gain classification information. In this study, we choose support vector machines (SVM) as $\pi_{A}$ to discriminate the meta-samples of cancer molecular patterns.

Theoretically, NPCA-based classification is rooted from a special nonnegative matrix factorization (NMF) that we propose in this study: the nonnegative principal component induced NMF. The principle of the NPCA-induced NMF can be briefed as follows. Let $X \in \Re^{d \times n}, d \ll n$, be a nonnegative matrix, which is a gene expression dataset with $d$ number of samples for $n$ number of genes in our context. Let $U \in \Re^{d \times d}$ be the corresponding nonnegative PC matrix for $X$, which is a near-orthogonal matrix before any further dimension selection. Projecting $X^{T}$ into the column space generated by $U$, we obtain the nonnegative projection $X^{T} U=P$. Since $U$ is a near-orthogonal matrix, we can view it as an orthogonal matrix to decompose the data matrix, i.e., $X^{T} \sim P U^{T}$, where $P$ is 
equivalent to the basis matrix $W$ and $U^{T}$ is equivalent to the feature matrix $H$ in the classic NMF $X^{T} \sim W H$ [5]. Unlike the general NMF, the basis matrix and the feature matrix in the NPCA-induced NMF both can be near-orthogonal.

The NPCA-based nonnegative matrix factorization can be explained alternatively. That is, each row of $U$ is a corresponding meta-sample of each biological sample of $X$ in the meta-gene space: $X_{i}^{T} \sim P U_{i}^{T}$. The meta-gene space $S=\operatorname{span}\left(p_{1}, p_{2} \cdots p_{r}\right), p_{i} \geq 0$ is a column space of the nonnegative basis matrix $P$, where each basis is a meta-gene. It is a purely additive space, where each variable can be represented as nonnegative linear combinations of meta-genes: $X_{i}^{T}=\sum_{j=1}^{r} U_{i j}^{T} P_{j}, 1 \leq r \leq d$.

Since we use SVM as $\pi_{A}$ in the NPCA-based classification, we brief NPCA-based SVM (NPCA-SVM) classification as follows. Considering gene expression data are naturally nonnegative data or can be converted to the corresponding nonnegative data easily, we conduct feature selection through nonnegative principal component analysis to obtain the low dimensional but data locality preserved meta-sample for each biological sample. Then, a SVM algorithm is employed to gain classification information from these meta-samples. To improve classification performance, we input the normalized metasamples, i.e., $U=U /\|U\|_{2}$, to the following SVM classification.

Since different robust levels of the prior knowledge from different training sets affect classification results for a classification algorithm, we conducted NPCA-SVM classifications under two types of data cross validations. The first is the $50 \%$ holdout cross validation with $\mathrm{N}=100$ times, i.e., 100 sets of training and test datasets generated by the $50 \%$ holdout cross validation for each dataset. The second is the leave one out cross validation $(L O O C V)$. To improve computing efficiency, the matrix $U$ is cached from previous trial and used as an initial point to compute the next trial principal component matrix in the classification.

\section{Experimental Results}

Table 1. Five Affymetrix oligonucleotide gene expression datasets

\begin{tabular}{lll}
\hline \multicolumn{1}{c}{ Dataset } & \#genes & \multicolumn{1}{c}{ \#samples } \\
\hline colon & 2,000 & 22 controls + 40 cancers \\
leukemia & 5,000 & 27 ALL +11 AML \\
medulloblastoma & 5,893 & 25 classic + 9 desmoplastic \\
hepatocellula carcinoma(HCC) & 7129 & 20 early intrahepatic recurrence \\
& & 40 non-early intrahepatic recurrence \\
glioma & 12,625 & 28 glioblastomas + 22 anaplastic oligodendrogliomas \\
\hline
\end{tabular}

We applied our NPCA-SVM algorithm for five bench-mark Affymetrix oligonucleotide gene expression datasets: colon, leukemia, medulloblastoma, hepatocellular carcinoma, and glioma $[6,7,8,9]$. Table 1 presents detailed information of the datasets. Without loss of generality, we choose two mostly used kernels in our NPCA-SVM algorithm: a general linear kernel and Gaussian ('rbf') kernel: $k(x, y)=(x \cdot y), \quad k(x, y)=\exp \left(-\|x-y\|^{2} / 2\right)$ 
[10]. We compared classification results from NPCA-SVM algorithm under the orthonormal control $\alpha=100$ with those from PCA-SVM and SVM algorithm under linear and Gaussian kernels for each of the five microarray datasets under 100 times (trials) of $50 \%$ holdout cross validations. The average classification rates, sensitivities and specificities and their corresponding standard deviations from these three algorithms are given in Table 2 .

Table 2. Average classification performances of three algorithms

\begin{tabular}{|c|c|c|c|}
\hline Dataset & $\begin{array}{c}\text { Average } \\
\text { Classifying } \\
\text { Rates }(\%)\end{array}$ & $\begin{array}{c}\text { Average } \\
\text { Sensitivity } \\
(\%)\end{array}$ & $\begin{array}{c}\text { Average } \\
\text { Specificity } \\
(\%)\end{array}$ \\
\hline \multicolumn{4}{|l|}{ Colon } \\
\hline npca-svm-linear & $89.77 \pm 4.79$ & $95.24 \pm 4.89$ & $81.76 \pm 11.24$ \\
\hline npca-svm-rbf & $88.90 \pm 5.33$ & $93.90 \pm 5.62$ & $81.10 \pm 11.60$ \\
\hline sum-linear & $46.10 \pm 11.25$ & $39.80 \pm 29.62$ & $55.14 \pm 31.59$ \\
\hline$s v m-r b f$ & $62.81 \pm 6.41$ & $100.0 \pm 0.0$ & $0.0 \pm 0.0$ \\
\hline pca-svm-linear & $75.90 \pm 7.96$ & $85.24 \pm 9.69$ & $61.73 \pm 16.80$ \\
\hline $\begin{array}{l}\text { pca-svm-rbf } \\
\text { Leukemia }\end{array}$ & $62.81 \pm 6.41$ & $100.0 \pm 0.0$ & $0.0 \pm 0.0$ \\
\hline npca-svm-linear & $96.11 \pm 5.57$ & $94.64 \pm 14.53$ & $99.16 \pm 2.69$ \\
\hline$n p c a-s v m-r b f$ & $91.84 \pm 8.51$ & $86.96 \pm 20.81$ & $94.25 \pm 7.04$ \\
\hline svm-linear & $44.21 \pm 22.08$ & $66.00 \pm 47.61$ & $34.00 \pm 47.61$ \\
\hline$s v m-r b f$ & $71.58 \pm 7.14$ & $0.0 \pm 0.0$ & $100.0 \pm 0.0$ \\
\hline pca-svm-linear & $95.32 \pm 6.97$ & $87.40 \pm 20.34$ & $99.26 \pm 2.46$ \\
\hline pca-svm-rbf & $71.58 \pm 7.14$ & $0.0 \pm 0.0$ & $100.0 \pm 0.0$ \\
\hline \multicolumn{4}{|l|}{ Medulloblostoma } \\
\hline npca-svm-linear & $86.18 \pm 9.47$ & $67.55 \pm 27.02$ & $94.09 \pm 9.17$ \\
\hline npca-svm-rbf & $81.76 \pm 9.80$ & $62.52 \pm 27.53$ & $90.68 \pm 11.21$ \\
\hline svm-linear & $35.88 \pm 20.30$ & $83.00 \pm 37.75$ & $17.00 \pm 37.75$ \\
\hline$s v m-r b f$ & $73.47 \pm 7.55$ & $0.0 \pm 0.0$ & $100.0 \pm 0.0$ \\
\hline pca-svm-linear & $80.82 \pm 8.47$ & $56.37 \pm 26.93$ & $90.95 \pm 8.38$ \\
\hline$p c a-s v m-r b f$ & $73.47 \pm 7.55$ & $0.0 \pm 0.0$ & $100.0 \pm 0.0$ \\
\hline \multicolumn{4}{|l|}{$\mathrm{HCC}$} \\
\hline npca-svm-linear & $88.37 \pm 5.16$ & $88.37 \pm 5.16$ & $86.73 \pm 5.36$ \\
\hline$n p c a-s v m-r b f$ & $86.73 \pm 5.36$ & $88.76 \pm 6.08$ & $84.62 \pm 16.66$ \\
\hline svm-linear & $41.07 \pm 15.55$ & $19.00 \pm 39.34$ & $81.00 \pm 39.34$ \\
\hline$s v m-r b f$ & $66.87 \pm 5.93$ & $0.0 \pm 0.0$ & $100.0 \pm 0.0$ \\
\hline pca-svm-linear & $60.93 \pm 7.90$ & $72.82 \pm 14.19$ & $39.53 \pm 17.70$ \\
\hline pca-svm-rbf & $66.87 \pm 5.93$ & $0.0 \pm 0.0$ & $100.0 \pm 0.0$ \\
\hline \multicolumn{4}{|l|}{ Glioma } \\
\hline npca-sum-linear & $91.24 \pm 5.11$ & $91.11 \pm 8.99$ & $91.83 \pm 6.68$ \\
\hline$n p c a-s v m-r b f$ & $90.20 \pm 5.40$ & $90.24 \pm 9.90$ & $90.66 \pm 7.69$ \\
\hline svm-linear & $49.56 \pm 8.22$ & $53.18 \pm 27.70$ & $45.72 \pm 28.72$ \\
\hline svm-rbf & $50.40 \pm 9.65$ & $18.00 \pm 38.61$ & $82.00 \pm 38.61$ \\
\hline pca-svm-linear & $72.68 \pm 6.75$ & $69.30 \pm 14.19$ & $76.17 \pm 11.64$ \\
\hline$p c a-s v m-r b f$ & $50.40 \pm 9.65$ & $18.00 \pm 38.61$ & $82.00 \pm 38.61$ \\
\hline
\end{tabular}

We have following observations from these classification results. 1. It is clear that the PCA-SVM, SVM algorithms suffer from the over-fitting problem under a Gaussian (' $r b f$ ') kernel. This can be found through the complementary results of the sensitivities and specificities for the five gene expression datasets obviously. 2 . There is no over-fitting 
problem, under a Gaussian (' $r b f$ ') kernel, for the NPCA-SVM algorithm; NPCA-SVM algorithm under a Gaussian kernel has the second best classification performance among all the results. 3. The classification results from our NPCA-SVM under a linear kernel have leading advantages over other two algorithms for all datasets.

Figure 1 shows the comparisons of the expectations of classification rates, sensitivities and specificities for the same 100 set of training and testing data for each gene expression dataset. Since PCA-SVM and SVM algorithms under a Gaussian kernel encountered the over-fitting problem, we did not include their sensitivities and specificities in the plot. It is obvious that NSPCA-SVM algorithm not only leads the PCA-SVM and SVM in the classification rates, sensitivities and specificities, but also it demonstrates the robust stability for the three measures. This can also be verified the relative small standard deviations for the three classifying performance measures of the NPCA-SVM classifications.
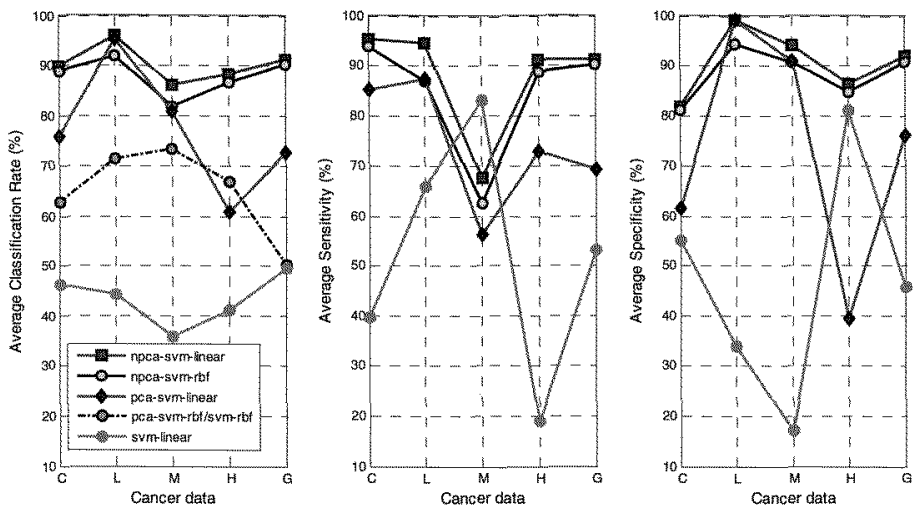

Figure 1. Comparisons on the average classification rates, sensitivities and specificities of the five gene expression datasets under NPCA-SVM, PCA-SVM and SVM classifications with linear and a Gaussian kernels. Each dataset is represented with its first letter in the figure. The performances of the NPCA-SVM algorithm are obviously superior to those of others in the scalar and stability.
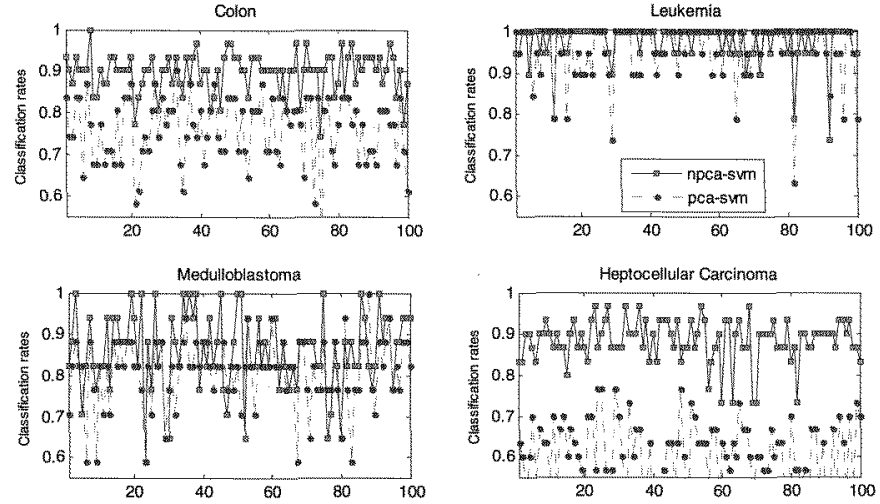

Figure 2. Comparisons on the classification rates of 100 trials for the four gene expression datasets under the NPCA-SVM and PCA-SVM classification with a linear kernel. The NPCA-SVM algorithm has the obviously leading or slightly better performances than the PCA-SVM under a linear kernel. 
Since the PCA-SVM algorithm under a linear kernel has the best classification results among the PCA-SVM and SVM classifications, we compare its performances with those of NPCA-SVM under a same kernel in Figure 2 for the first four datasets. It is easy to find that our NPCA-SVM algorithm has achieved obviously leading performances for colon, medulloblastoma and heptocellular carcinoma datasets under a linear kernel, when compared with the PCA-SVM algorithm. Our NPCA-SVM algorithm also achieves slightly better results for the leukemia dataset than the PCA-SVM algorithm under a linear kernel. According to our experimental results, the NPCA-SVM classification results from Glioma dataset also strongly demonstrated its leading advantages over the PCA-SVM algorithm under a linear kernel.

\subsection{Classification results comparisons with those of other algorithms}

It is desirable to compare the nonnegative principal component analysis based SVM algorithm (NPCA-SVM) with other classification algorithms to further verify its superiority. In this section, we compare the classification performances of our NPCASVM algorithm with those of other five classification algorithms. These five algorithms include $k$-nearest neighbor $(\mathrm{k}-\mathrm{NN})$, linear discriminant analysis under principal component analysis (PCA-LDA) and three nonlinear feature selection based SVM classification algorithms: SVM classifications under kernel principal component analysis (KPCA-SVM); nonnegative matrix factorization based SVM (NMF-SVM) and SVM classifications under locally linear embedding (LLE-SVM). Details about these three feature selection algorithms can be found in $[5,11,12]$.

The k-NN and PCA-LDA algorithms both are widely used algorithms in microarray data classifications. The $\mathrm{k}-\mathrm{NN}$ is a simple Bayesian inference method. It determines the class type of a sample interested based on the class belongs of its nearest neighbors, which are measured by correlation, Euclidean or other distances. In PCA-LDA classifications, it conducts PCA processing for training samples and projects testing samples in the subspace spanned by the principal components of the training samples at first. Then, a linear discriminant analysis (LDA) is used to classify projections of the testing samples, which is equivalent to solving a generalized eigenvalue problem [2].

The three nonlinear feature selection based SVM classification algorithms conduct SVM classification for the meta-samples in the space generated by corresponding feature selection algorithms respectively. For instance, KPCA-SVM conducts classification for the projections of testing data in the space spanned by the PCs of training data, obtained by performing PCA in a kernel space; LLE-SVM conducts classification for the metasamples of input biological samples, which are the low dimensional and neighborhood preserving embedding of the original high dimensional data. For convenience, we brief the NMF-SVM classification algorithm as follows.

The NMF-SVM algorithm is to decompose the nonnegative gene expression cancer data $X \in \Re^{n \times m}$ into the product of two nonnegative matrices: $X \sim W H$, under a rank $r$ with the least reconstruction error. The matrix $W \in \Re^{n \times r}$ is called a basis matrix. Its column space sets up a new coordinate system for $X$; the matrix $H \in \Re^{r \times m}$ is called a feature matrix. It stores the new coordinate values for each variable of $X$ in the new space. Then, a SVM algorithm is used to classify the corresponding meta-sample of each sample 
in the gene expression matrix $X$, which is the corresponding column in the feature matrix $H$.

For each dataset, we still use previous 100 trial of training and testing data from $50 \%$ holdout cross validations in classifications. Table 3 shows the average sensitivities and specificities and their corresponding standard deviations for four algorithms.

Table 3. Average classification performances of NMF/LLE-SVM, PCALDA and $k-N N$

\begin{tabular}{|c|c|c|c|}
\hline Dataset & $\begin{array}{l}\text { Average } \\
\text { Classifying } \\
\text { Rates (\%) } \\
\end{array}$ & $\begin{array}{l}\text { Average } \\
\text { Sensitivity } \\
(\%) \\
\end{array}$ & $\begin{array}{l}\text { Average } \\
\text { Specificity } \\
(\%) \\
\end{array}$ \\
\hline \multicolumn{4}{|l|}{ Colon } \\
\hline Nmf-svm-linear & $84.03 \pm 6.31$ & $89.28 \pm 6.81$ & $76.68 \pm 13.70$ \\
\hline$N m f-s v m-r b f$ & $74.42 \pm 7.89$ & $86.98 \pm 9.62$ & $55.23 \pm 16.72$ \\
\hline knn-euclidean & $78.03 \pm 7.57$ & $90.38 \pm 7.15$ & $58.65 \pm 7.15$ \\
\hline knn-correlation & $80.52 \pm 8.78$ & $93.31 \pm 4.36$ & $61.61 \pm 18.96$ \\
\hline pca-lda & $86.39 \pm 5.64$ & $89.37 \pm 6.74$ & $81.65 \pm 10.91$ \\
\hline lle-sum-linear & $83.71 \pm 5.47$ & $93.96 \pm 4.85$ & $67.04 \pm 12.00$ \\
\hline $\begin{array}{l}\text { lle-svm-rbf } \\
\text { Leukemia }\end{array}$ & $73.81 \pm 7.85$ & Leukemia & $58.40 \pm 19.16$ \\
\hline Nmf-svm-linear & $92.16 \pm 7.19$ & $77.79 \pm 21.69$ & $98.46 \pm 3.89$ \\
\hline$N m f-s v m-r b f$ & $88.89 \pm 8.59$ & $74.87 \pm 23.85$ & $95.12 \pm 6.45$ \\
\hline knn-euclidean & $91.32 \pm 7.75$ & $76.72 \pm 23.95$ & $98.09 \pm 3.88$ \\
\hline knn-correlation & $93.05 \pm 5.93$ & $83.42 \pm 17.08$ & $97.79 \pm 4.36$ \\
\hline pca-lda & $94.21 \pm 6.79$ & $81.98 \pm 19.62$ & $100.00 \pm 0.0$ \\
\hline lle-svm-linear & $95.00 \pm 3.03$ & $90.93 \pm 12.35$ & $96.75 \pm 4.00$ \\
\hline lle-svm-rbf & $89.11 \pm 7.71$ & $81.76 \pm 17.30$ & $92.59 \pm 9.58$ \\
\hline \multicolumn{4}{|l|}{ Medulloblostoma } \\
\hline Nmf-svm-linear & $81.76 \pm 9.70$ & $64.95 \pm 25.56$ & $88.22 \pm 10.87$ \\
\hline$N m f-s v m-r b f$ & $82.18 \pm 8.98$ & $58.98 \pm 27.03$ & $91.98 \pm 10.87$ \\
\hline knn-euclidean & $76.59 \pm 10.51$ & $24.22 \pm 29.61$ & $98.66 \pm 4.79$ \\
\hline knn-correlation & $79.12 \pm 10.08$ & $50.22 \pm 27.97$ & $91.66 \pm 11.22$ \\
\hline pca-lda & $81.24 \pm 9.59$ & $58.55 \pm 28.92$ & $92.21 \pm 8.79$ \\
\hline lle-svm-linear & $76.00 \pm 10.47$ & $53.34 \pm 29.50$ & $85.60 \pm 12.36$ \\
\hline \multicolumn{4}{|l|}{ HCC } \\
\hline Nmf-svm-linear & $61.30 \pm 8.91$ & $71.17 \pm 13.47$ & $43.47 \pm 16.67$ \\
\hline$N m f-s v m-r b f$ & $58.67 \pm 8.49$ & $79.52 \pm 9.21$ & $22.83 \pm 13.14$ \\
\hline knn-euclidean & $61.83 \pm 7.92$ & $79.54 \pm 14.73$ & $25.84 \pm 19.58$ \\
\hline$k n n$-correlation & $63.10 \pm 7.80$ & $81.55 \pm 11.18$ & $27.86 \pm 16.15$ \\
\hline pca-lda & $60.87 \pm 7.82$ & $72.88 \pm 14.42$ & $39.47 \pm 17.08$ \\
\hline lle-svm-linear & $62.77 \pm 7.36$ & $91.78 \pm 13.87$ & $5.81 \pm 12.93$ \\
\hline lle-svm-rbf & $66.83 \pm 5.87$ & $99.96 \pm 0.42$ & $0.0 \pm 0.0$ \\
\hline \multicolumn{4}{|l|}{ Glioma } \\
\hline Nmf-svm-linear & $74.40 \pm 8.04$ & $74.54 \pm 11.10$ & $74.19 \pm 13.53$ \\
\hline Nmf-svm-rbf & $70.40 \pm 5.40$ & $51.87 \pm 13.61$ & $84.07 \pm 7.79$ \\
\hline knn-euclidean & $46.80 \pm 8.76$ & $47.85 \pm 16.66$ & $47.63 \pm 15.84$ \\
\hline knn-correlation & $74.56 \pm 7.66$ & $74.14 \pm 13.12$ & $76.24 \pm 11.90$ \\
\hline pca-lda & $73.44 \pm 6.93$ & $70.82 \pm 13.91$ & $76.41 \pm 12.47$ \\
\hline lle-svm-linear & $73.84 \pm 8.82$ & $73.00 \pm 14.39$ & $74.93 \pm 13.61$ \\
\hline lle-svm-rbf & $65.92 \pm 12.19$ & $47.22 \pm 26.80$ & $84.97 \pm 22.50$ \\
\hline
\end{tabular}


In the $\mathrm{k}-\mathrm{NN}$ algorithm, the distance measure was chosen as the correlation or Euclidean distance and the number of nearest neighbors was selected from 2 to 7 . In the LLE-SVM classification, we selected embedding dimensionalities from 2 to 20 . In the NMF-SVM classification, the matrix decomposition rank in the NMF was selected from 2 to 18. The kernel function in SVM is still selected as a linear or Gaussian kernel. The final average classification rate for a dataset under each algorithm is selected as the best average classification rate among all possible cases.

In the KPCA-SVM algorithm, there are two kernel functions: one is kernel function $k_{1}(x, y)$ in kernel PCA and another is kernel $k_{2}(x, y)$ in the following SVM algorithm. When $k_{1}(x, y)$ is a linear kernel, then kernel PCA is just original PCA and KPCA-SVM has the same performance as the previous PCA-SVM algorithm; when $k_{i}(x, y)$ is a Gaussian kernel, we have found that KPCA-SVM encounters the over-fitting problem for all five datasets no matter $k_{2}(x, y)$ is a linear or Gaussian kernel.

From our experimental results, we have found that the NMF-SVM algorithm generally has better classification results under a linear kernel than a Gaussian kernel, although the NMF-SVM classification under a linear kernel has slightly better performance than that under a Gaussian kernel for the medulloblastoma dataset. NMFSVM also overcomes the over-fitting problem under a Gaussian kernel. This is because that the meta-samples are from the space generated from nonnegative matrix factorization, is also a purely additive space. We have found LLE-SVM algorithm generally has better classification performances under a linear kernel than a Gaussian kernel. However, it still cannot avoid the over-fitting problem under a Gaussian kernel, because this manifold learning based algorithm encountered the over-fitting for medulloblastoma and heptocellular carcinoma $(H C C)$ datasets. It is also easy to see that the $\mathrm{k}-\mathrm{NN}$ classification under the correlation distance has the advantages over the Euclidean distance for the five cancer datasets. However, from these results, we can observe that the performances of all these four algorithms still can not compete with those of NPCA-SVM algorithm for the five microarray datasets, under the 100 trials of $50 \%$ holdout cross validations.
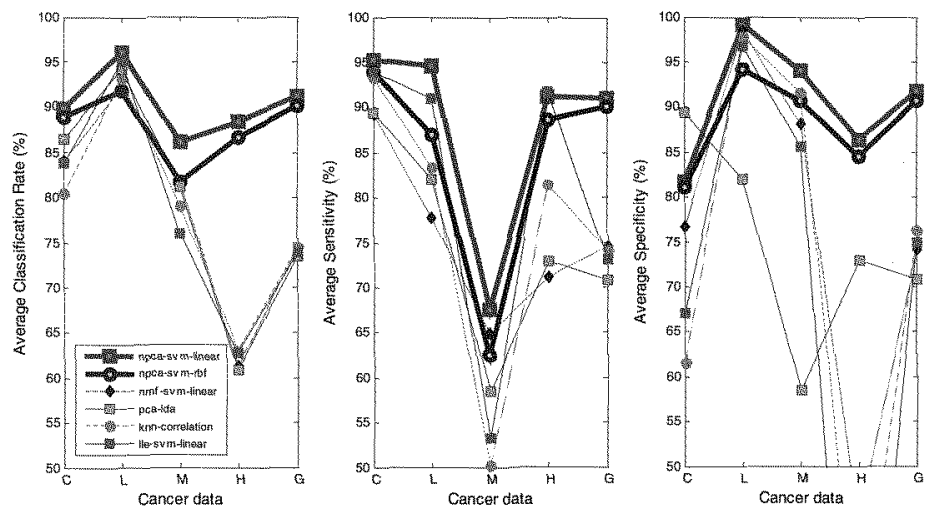

Figure 3. Comparisons on the average classification rates, sensitivities and specificities of the five gene expression datasets under the NPCA-SVM, NMF-SVM, LLE-SVM, PCA-LDA, KNN classifications. Each cancer dataset is represented with its first letter in Figure. The NPCA-SVM algorithm achieves the stably leading classification performances for the five datasets. 
Figure 3 shows the comparisons on the average classification rates, sensitivities and specificities of the NPCA-SVM algorithm under the linear and Gaussian kernel with those of other four classification algorithms: NMF-SVM, LLE-SVM, PCA-LDA, k-NN, for the five gene expression datasets. We can observe that the average classification rates, sensitivities and specificities of our NPCA-SVM algorithms are clearly superior to those of others in a stable pattern. Alternatively, the curves of average classification rates, sensitivities and specificities from the other algorithms have relatively large oscillations, for the five microarray datasets.

We also compared our NPCA-SVM algorithm with six other algorithms: PCA-SVM, SVM, NMF-SVM, LLE-SVM, k-NN and PCA-LDA for the five datasets under the leave one out cross validations ( $L O O C V$ ). The classification rate for each algorithm under $\angle O O C V$ is the ratio between the total numbers of correctly classified samples over the number of total samples. Figure 4 demonstrates that the NPCA-SVM algorithm, under linear and Gaussian kernels, have strongly leading performances over the other six algorithms for the five cancer datasets under $L O O C V$ on classification rates. In our plot, we selected the classification results from NMF-SVM, LLE-SVM algorithms under linear kernels for their better performances than Gaussian kernels. For the same reason, we selected k-NN classification results under correlation distances instead of Euclidean distances. We can observe that only the PCA-SVM algorithm under a linear kernel has achieved a comparable result at leukemia dataset, compared with those of our NPCASVM algorithm. For other four gene expression datasets, the classification results from the NPCA-SVM algorithm, under linear and Gaussian kernels, are obviously superior to those of the other six algorithms. Such a result is consistent with the previous results under the 50\% holdout cross validations. Under a Gaussian kernel, algorithms PCA-SVM, SVM, LLE-SVM all suffer from the over-fitting problem. However, just as before, there is no over-fitting problem in the NPCA-SVM algorithm.

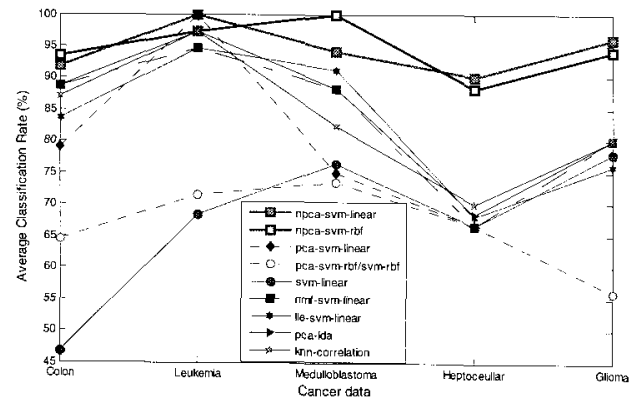

Figure 4. Comparisons of the classification rates of seven algorithms: NPCA-SVM, PCA-SVM, SVM, NMFSVM, LLE-SVM, PCA-LDA and k-NN for five cancer datasets, under the leave one out cross validations ( $L O O C V$ ). The NPCA-SVM algorithm has the obviously leading advantages over other six classification algorithm for the five gene expression datasets.

\section{Discussions and Conclusions}

In this study, we present a novel nonnegative principal component analysis (NPCA) algorithm and apply it in the gene expression data classification. We have demonstrated that NPCA-SVM algorithm has obviously leading advantages over other seven 
classification algorithms in the cancer pattern classification for five microarray datasets under the $50 \%$ hold-out and leave one out cross validations. The general over-fitting problem associative with SVM based classification in gene expression data under a Gaussian kernel is also overcome in our algorithm.

From the nonnegative principal component analysis, we can develop a family of NPCA-induced statistical learning algorithms by applying NPCA as a feature selection algorithm before a classification or clustering algorithm. For example, NPCA-based Fisher discriminant analysis (NPCA-FDA), etc. Alternatively, since NPCA-SVM is a more robust high-performance classifier than the general SVM and k-NN classifiers, it can replace the popular SVM and k-NN classifiers used in the cancer biomarker identification to capture oncology genes. In the following work, we plan to investigate applications of NPCA-based classification algorithms in the SNP array, exon-array and proteomics data and related biomarker discovery.

\section{References}

[1] Pochet N., De Smet F., Suykens J.A.K. and De Moor B.L.R., Systematic benchmarking of microarray data classification: assessing the role of non-linearity and dimensionality reduction, Bioinformatics, 20(17), 3185-3195, 2004.

[2] Lilien, R. and Farid, H., Probabilistic Disease Classification of Expressiondependent Proteomic Data from Mass Spectrometry of Human Serum, Journal of Computational Biology, 10(6), 925-946, 2003.

[3] Gao, Y. and Church, G., Improving molecular cancer class discovery through sparse nonnegative matrix factorization, Bioinformatics, 21(21), 3970-3975, 2005.

[4] Han, X., Cancer molecular pattern discovery by subspace consensus kernel classification, Computational Systems Bioinformatics, Proceedings of the Conference CSB 2007, 6:55-65, 2007.

[5] Daniel D. Lee and H. Sebastian Seung., Learning the parts of objects by nonnegative matrix factorization. Nature, 401, 788-791, 1999.

[6] Alon,A.,et al., Broad patterns of gene expression revealed by clustering analysis of tumor and normal colon tissues probed by oligonucleotide arrays. Proc. Natl Acad. Sci. USA, 96, 6745-6750. 1999.

[7] Brunet, J., Tamayo, P., Golub, T. and Mesirov., J., molecular pattern discovery using matrix factorization. Proc. Natl Acad. Sci. USA, 101(12), 4164-4169, 2004.

[8] Iizuka,N., et al., Oligonucleotide microarray for prediction of early intrahepatic recurrence of hepatocellular carcinoma after curative resection, The Lancet, 361,923-929, 2003.

[9] Nutt,C.L., et al., Gene expression-based classification of malignant gliomas correlates better with survival than histological classification, Cancer Research, 63(7), 1602-1607, 2003.

[10] Vapnik,V.N., Statistical Learning Theory, John Wiley \& Sons, New York,1998.

[11] Schölkopf, B., Smola, A. J., and Müller, K.-R., Nonlinear component analysis as a kernel eigenvalue problem, Neural Computation, 10, 1299-1319, 1998.

[12] Roweis, S. and Saul, L., Nonlinear dimensionality reduction by locally linear embedding, Science, v.290 no.5500, 2323-2326, 2000. 